\title{
Effect of chitin and chitosan hexamers on growth and photosynthetic characteristics of wheat seedlings
}

\author{
K.C. $\mathrm{LI}^{*, * *,+}$, X.Q. ZHANG ${ }^{*, * * *}$, Y. YU”, R.E. XING ${ }^{*, * *}$, S. LIU*, and P.C. LI ${ }^{*, * *,+}$ \\ Key Laboratory of Experimental Marine Biology, Center for Ocean Mega-Science, Institute of Oceanology, \\ Chinese Academy of Sciences, 266071 Qingdao, China* \\ Laboratory for Marine Drugs and Bioproducts of Qingdao National Laboratory for Marine Science \\ and Technology, 266237 Qingdao, China** \\ School of Marine Sciences, Ningbo University, 315211 Ningbo, China ${ }^{* * *}$ (current address) \\ Marine Science and Engineering College, Qingdao Agricultural University, 266109 Qingdao, China
}

\begin{abstract}
In this study, two homogeneous chitin and chitosan hexamers and one partially acetylated chitosan hexamer were used to investigate the degree of acetylation (DA) effects of chitooligosaccharides on the growth and photosynthesis of wheat seedlings. Both chitin and chitosan hexamers showed a significant promoting effect on the growth and photosynthesis of wheat seedlings but the positive effect depended on their DA. The homogeneous chitosan hexamers $\left[(\mathrm{GlcN})_{6}\right]$ exhibited the optimal activity compared to other samples. Seven days after $(\mathrm{GlcN})_{6}$ treatment, the growth parameters of wheat seedlings were all significantly enhanced including fresh mass, dry mass, and length of both shoots and roots, and the contents of soluble sugar were increased by $22.8 \%$. Additionally, $(\mathrm{GlcN})_{6}$ could significantly promote (by $\left.90.0 \%\right)$ the photosynthetic rate of wheat seedlings. Both Rubisco and fructose-1,6-bisphosphatase activity of photosynthetic carbon metabolism was also found to be significantly improved by $(\mathrm{GlcN})_{6}$ treatment.
\end{abstract}

Additional key words: biostimulator; chitohexaose; partial acetylation; plant growth promotion; structure-function relationship.

\section{Introduction}

Chitin is the second most abundant polysaccharide in nature, consisting of $\beta(1 \rightarrow 4)$-linked N-acetyl-D-glucosamine (GlcNAc, A) residues. Chitin exists mainly as a structural component in the cell walls of fungi and yeasts and in the exoskeletons of insects, nematodes, and arthropods (Rinaudo 2006). Chitosan, a product of the deacetylation reaction of chitin, is a heterogeneous polysaccharide comprised of GlcNAc and D-glucosamine (GlcN, D) residues. Both chitin and chitosan are nontoxic, biocompatible, and biodegradable, and exhibit a variety of interesting physicochemical and biological properties. The unique combination of these characteristics makes chitin and chitosan particularly useful for numerous applications in the fields of agriculture, food, and medicine (Muzzarelli 2010, Domard 2011).

Chitooligosaccharide (COS) is a hydrolyzed product of chitosan. Compared with chitosan, COS has much lower molecular mass $(\mathrm{Mw})$ and higher solubility in aqueous solutions, and possesses diverse special biological functions, such as anticancer (Huang et al. 2006), anti-inflammatory (Fernandes et al. 2010), immunityenhancing activity (Xing et al. 2017), and so on. In agriculture, COSs are regarded as a potent pathogenassociated molecular pattern (PAMP) and can elicit plant innate immunity by inducing a set of defense reactions, such as depolarization of membrane potential, production of reactive oxygen species and phytoalexin synthesis (Kuchitsu et al. 1995, Yin et al. 2016). Apart from biotic stress, it was also reported that COS could enhance the abiotic stress tolerance of plants, such as drought (Dzung et al. 2011), chilling (Zou et al. 2017), salt (Jabeen and Ahmad 2013, Zhang et al. 2017a), and heavy metal ion stress (Zong et al. 2017a). Furthermore, COS has attracted increasing interest as a potential biostimulator to promote

$\overline{\text { Received } 16 \text { September } 2019}$, accepted 28 February 2020.

${ }^{+}$Corresponding author; e-mail: 1kc@qdio.ac.cn (K.C. Li), pcli@qdio.ac.cn (P.C. Li)

Abbreviations: A6 - chitin hexamer; $C_{\mathrm{i}}$ - intercellular $\mathrm{CO}_{2}$ concentration; $\mathrm{COS}$ - chitooligosaccharide; D6 - chitosan hexamer; DA - degree of acetylation; DP - degree of polymerization; $E$ - transpiration rate; FBPase - fructose-1,6-bisphosphatase; FM - fresh mass; $F_{v} / F_{m}-$ maximum quantum yield of PSII photochemistry; GlcN - D-glucosamine; $(\mathrm{GlcN})_{6}$ - chitosan hexamer; GlcNAc $\mathrm{N}$-acetyl-D-glucosamine; (GlcNAc) 6 - chitin hexamer; $g_{\mathrm{s}}$ - stomatal conductance; Mw - molecular mass; NAD6 - N-acetylated chitosan hexamer; NPQ - nonphotochemical quenching coefficient; PAMP - pathogen-associated molecular pattern; $P_{\mathrm{N}}$ - photosynthetic rate; $\mathrm{q}_{\mathrm{L}}$ - photochemical quenching coefficient; $\Phi_{\text {PSII }}$ - effective quantum yield of PSII photochemistry.

Acknowledgements: The study was supported by the National Natural Science Foundation of China (41976096, 41406086), National Key R\&D Program of China (2018YFC0311305, 2019YFD0902105), and Key Research and Development Program of Shandong Province (2019YYSP028) 
plant growth. It has been recently reported that COS could stimulate seed germination and tissue growth of orchid (Nge et al. 2006, Kananont et al. 2010), enhance the photosynthesis of maize (Khan et al. 2002), promote mineral nutrient uptake of bean (Chatelain et al. 2014), and increase the yield of chili and wheat (Wang et al. 2015, Dzung et al. 2017).

The effectiveness of COS in various bioactivities was significantly correlated with its chemical structure. Degree of acetylation (DA) is one of the most important structural parameters affecting the biological properties of COS. Wang et al. (2007) reported that the antiangiogenic activity of $\mathrm{N}$-acetylated COS with DA $81 \%$ was significantly stronger than that of the oligosaccharides with DA 5\%. Lieder et al. (2012) investigated the effect of COS on expansion and differentiation of human mesenchymal stem cells and found that the oligomers with higher DA showed stronger effects. Kumar et al. (2005) reported that the antibacterial activity of hetero-COS was relied on their GlcN/GlcNAc ratio and a lower value of DA would be beneficial for its antibacterial activity. Therefore, it seemed that the structure-function relationship between DA of COS and various bioactivities could be different.

In agriculture, COS has also different functions with a primary factor of DA difference. Vander et al. (1998) reported that partially $\mathrm{N}$-acetylated COS showed stronger elicitor activity than that of homooligomers of either GlcNAc or GlcN in wheat leaves. The activity of COS enhancing plant tolerance to abiotic stress was also verified to be closely related with its DA (Zou et al. 2015). Concerning plant growth regulation, both chitin and chitosan oligomers were reported to show positive effect (Zhang et al. 2016, Winkler et al. 2017). However, to the best of our knowledge, few comparison reports on the plant growth promotion among COSs with different DA were presented and the DA effects of COS on plant growth under natural conditions were still unknown.

Accordingly, this work was implemented using three COSs with well-defined DA, including two homogeneous hexamers (GlcNAc) 6 and $(\mathrm{GlcN})_{6}$ and one partially acetylated chitosan hexamer. The DA effects of COS on the growth and photosynthesis of wheat seedlings were investigated. These results were fundamental to the study of the action mechanism of COS in promoting plant growth and their application as plant growth regulators.

\section{Materials and methods}

Materials: Chitosan hexamer $\left[(\mathrm{GlcN})_{6}, \mathrm{D} 6, \geq 95 \%\right.$ ], was separated from a fully deacetylated COS by ion-exchange chromatography as reported by our previous study ( $\mathrm{Li}$ et al. 2013). Chitin hexamer [(GlcNAc) $6, \mathrm{~A} 6, \geq 95 \%$ ] was purchased from Qingdao Qunheng Biotech Co., Ltd. (Qingdao, China). Sephadex G15 was purchased from GE Healthcare (USA). Acetic anhydride and other chemical reagents were of analytic grade without further purification.

Preparation and characterization of partially N-acetylated chitosan hexamer (NAD6): The selective N-acetylation of chitosan hexamer was performed according to previous method (Li et al. 2017). Briefly, $30 \mathrm{mg}$ of chitosan hexamer was dissolved in $5 \mathrm{~mL}$ of a methanol/ water $(50: 50, v / v)$ solution. Acetic anhydride $(30 \mu \mathrm{L})$ was added stoichiometrically to the chitosan hexamer solution under magnetic stirring at room temperature for $1 \mathrm{~h}$. Subsequently, the resulting solution was concentrated and further purified by a Sephadex G15 column $(1.6 \times$ $100 \mathrm{~cm}$ ) using water as eluent. The purified fraction was lyophilized and referred as NAD6.

The DA of $\mathrm{N}$-acetylated chitosan hexamer was determined by ${ }^{1} \mathrm{H}$ NMR spectroscopy. ${ }^{1} \mathrm{H}$ NMR spectrum was recorded on a JEOL JNM-ECP600 spectrometer (JEOL, Tokyo, Japan) under a static magnetic field of $600 \mathrm{MHz}$ at room temperature. NAD6 was dissolved in $\mathrm{D}_{2} \mathrm{O}$.

Plant material and treatments: Wheat (Triticum aestivum L. cv. Jimai 22) seeds were used in the present study. The growth conditions of wheat seedlings were set according to Zhang et al. (2016). Firstly, wheat seeds were surfacesterilized with a $1 \%$ sodium hypochlorite solution and then rinsed several times with distilled water. Subsequently, 30 seeds were transferred to a Petri dish with moist gauze for germination at $25^{\circ} \mathrm{C}$ for $24 \mathrm{~h}$ in the dark. Then, germinated seeds were individually transferred to Petri dishes with nylon mesh and each Petri dish contained 30 seeds. Wheat seedlings were cultivated with Hoagland solution in a growth incubator. The LED $(Y Z 28 R R 16 / G)$ light intensity was $800 \mu \mathrm{mol}\left(\right.$ photon) $\mathrm{m}^{-2} \mathrm{~s}^{-1}$ with a day/night cycle of $14 / 10 \mathrm{~h}$ at $25 / 15^{\circ} \mathrm{C}$, respectively, and the relative humidity was controlled at 70\%. Hoagland solution was renewed every day. When the second functional leaf of wheat seedlings was fully expanded, the plants were randomly divided into four groups and each group contained three biological replicates. The control group was sprayed with distilled water and the oligosaccharidestreated groups were sprayed with $15 \mathrm{mg} \mathrm{L}^{-1}$ of A6, NAD6 or D6, respectively. Tween $20(0.1 \%)$ was added to all the samples.

Growth parameters: Wheat seedlings of each group were harvested on the $7^{\text {th }}$ day after chitin and chitosan hexamers treatments. The growth parameters of wheat seedlings were determined including shoot length, root length, and fresh mass (FM) and dry mass (DM). For the determination of shoot and root fresh mass, the plant was cut between the hypocotyl and root. Before the fresh mass was determined, the root was padded dry with absorbent paper. As for the determination of dry mass, plant materials were dried at $105^{\circ} \mathrm{C}$ for $3 \mathrm{~h}$.

Chlorophyll (Chl) content was analyzed by spectrophotometry following the method of Lichtenthaler and Wellburn (1983). Seven days after chitin and chitosan hexamers treatment, Chl was extracted with $95 \%$ ethanol. Absorbance values of the extracted solutions containing Chl were measured at 665 and $649 \mathrm{~nm}$, respectively (TU1810 UV-visible spectrophotometer, Beijing Puxi, China). The Chl $a$, Chl $b$, and total Chl $(a+b)$ contents in the leaf tissues of wheat seedlings were then calculated from the measured absorbance values according to the 
following equations and expressed as $\mathrm{mg}(\mathrm{Chl}) \mathrm{g}^{-1}$ (fresh mass):

Chl $a=13.95 \mathrm{~A}_{665}-6.88 \mathrm{~A}_{649}$

Chl $b=24.96 \mathrm{~A}_{649}-7.32 \mathrm{~A}_{665}$

$\operatorname{Chl}(a+b)=6.63 \mathrm{~A}_{665}+18.08 \mathrm{~A}_{649}$

Measurement of photosynthetic characteristics: Photosynthetic rate $\left(P_{\mathrm{N}}\right)$, transpiration rate $(E)$, stomatal conductance $\left(g_{\mathrm{s}}\right)$, and intercellular $\mathrm{CO}_{2}$ concentration $\left(C_{\mathrm{i}}\right)$ were measured with a portable photosynthesis system (L.MAN-LCPro-SD, BioScientific Ltd., UK). The gas-flow rate was $200 \mu \mathrm{mol} \mathrm{s}{ }^{-1}$, PPFD density was $800 \mu \mathrm{mol} \mathrm{m}^{-2} \mathrm{~s}^{-1}$, and atmospheric $\mathrm{CO}_{2}$ concentration was $415 \pm 5 \mu \mathrm{mol}$ $\mathrm{mol}^{-1}$.

Chl fluorescence parameters: Chl fluorescence was measured using a portable fluorometer (PAM-2100, Walz, Germany). For each plant, the fluorescence was analyzed from the upper surface of the second functional leaf. All parameters were measured after dark adaptation for $30 \mathrm{~min}$, including the maximum quantum yield of PSII photochemistry $\left(\mathrm{F}_{\mathrm{v}} / \mathrm{F}_{\mathrm{m}}\right)$, effective quantum yield of PSII photochemistry $\left(\Phi_{\mathrm{PSII}}\right)$, photochemical quenching coefficient $\left(\mathrm{q}_{\mathrm{L}}\right)$, and nonphotochemical quenching coefficient (NPQ) (Roháček 2002).

Soluble sugar: Seven days after chitin and chitosan hexamers treatments, soluble sugar content was measured by phenol-sulfuric acid reaction (Zou et al. 2015). Briefly, $0.3 \mathrm{~g}$ of fresh leaf samples were cut up and heated at $100^{\circ} \mathrm{C}$ for $30 \mathrm{~min}$ in $5 \mathrm{~mL}$ of distilled water. The resulting extract was filtrated and diluted 5-fold for determination. Diluted sample of $500 \mu \mathrm{L}$ was mixed with $1 \mathrm{~mL}$ of $5 \%$ phenol and then $5 \mathrm{~mL}$ of sulfuric acid was added quickly. The absorbance was read at $485 \mathrm{~nm}$ and the soluble sugar concentration was quantified using a glucose standard curve (TU1810 UV-visible spectrophotometer, Beijing Puxi, China).

Enzyme assays: Seven days after chitin and chitosan hexamers treatments, the second functional leaves were pulverized in liquid $\mathrm{N}_{2}$ with a chilled mortar and pestle and then used to determine the enzyme activities. Regarding the determination of Rubisco (EC 4.1.1.39) activity, $0.1 \mathrm{~g}$ of plant tissue was homogenized in an extraction buffer containing $100 \mathrm{mM}$ HEPES-KOH (pH 8.0), $1 \mathrm{mM} \mathrm{MgCl}_{2}$, $1 \mathrm{mM}$ EDTA, $2 \mathrm{mM}$ dithiothreitol (DTT), and $1 \mathrm{mM}$ phenylmethylsulfonyl fluoride (PMSF); the total Rubisco enzyme was extracted by ultrasonic treatment in an ice bath. After centrifugation, the Rubisco activities were measured with a spectrophotometer at $340 \mathrm{~nm}$ (TU1810 UV-visible spectrophotometer, Beijing Puxi, China), and one unit of enzyme activity was defined as 1 nmol of $\mathrm{NADH}$ oxidation per min at $25^{\circ} \mathrm{C}$ (Sharkey et al. 1991).

As for the determination of fructose-1,6-bisphosphatase (FBPase, EC 3.1.3.11) activity, the total enzyme was similarly extracted under ultrasonic conditions and the FBPase activities were assayed by the method reported by Hurry et al. (1995). Results for the FBPase activities were presented as $1 \mathrm{nmol}$ of NADPH generation per min at $25^{\circ} \mathrm{C}$.

Statistical analysis: Each test was performed in triplicate and the data were expressed as means \pm SD of three independent replicates. Statistical analyses were conducted using analysis of variance (ANOVA) analysis and Duncan's multiple test $(P<0.05)$ by SPSS 22.0 software.

\section{Results}

DA determination of partially $\mathbf{N}$-acetylated chitosan hexamer (NAD6): In order to investigate better the DA effects of chitooligosaccharides on plant growth promotion, a partially $\mathrm{N}$-acetylated chitosan hexamer (NAD6) was prepared by a reacetylation reaction of chitosan hexamer (D6), and further used for plant treatment experiments to make a comparison with two homogeneous hexamers $(\mathrm{GlcNAc})_{6}$ and $(\mathrm{GlcN})_{6}$. The DA of the prepared partially $\mathrm{N}$-acetylated chitosan hexamer was determined by ${ }^{1} \mathrm{HNMR}$ spectroscopy (Fig. 1). The average DA of NAD6 was calculated using both signal areas of $\mathrm{H}-2$ protons of GlcN units $\left(\mathrm{A}_{\mathrm{GlN} \mathrm{H}-2}\right)$ and acetyl protons of GlcNAc units $\left(\mathrm{A}_{\mathrm{CH} 3}\right)$ as reported by Trombotto et al. (2008). In this case, the DA of NAD6 used for plant treatment experiments was $32.2 \%$.

Plant growth parameters: The three hexamer samples with various DA showed different effects on the growth parameters of wheat seedlings (Fig. 2). Compared with the control group, all of A6, NAD6, and D6 could obviously promote the shoot biomass accumulation. The shoot fresh masses of A6, NAD6, and D6 treatment groups increased by $15.9,19.3$, and $25.9 \%$, respectively, and correspondingly, the shoot dry masses increased by $17.5,25.9$, and $30.0 \%$, respectively. D6 showed a stronger promoting effect on the shoot biomass accumulation of wheat seedlings than that of A6. However, the shoot length data did not show significant difference between three hexamer-treated groups. For roots, the enhancement of fresh mass, dry mass, and length in seedlings treated by D6 and NAD6 were also higher than those of A6-treated group. Compared with the control group, A6 treatment showed a relative increase of the root biomass accumulation in wheat seedlings (root fresh mass and dry mass) but with no significant promotion.

Soluble sugar content: All three hexamer samples could obviously promote the soluble contents of wheat seedlings compared with the control group (Fig. 3). After exogenous treatments with A6, NAD6, and D6, the soluble sugar contents of wheat seedlings increased by $8.2,21.5$, and $22.8 \%$, respectively, while the promoting effect of A6 was less intensive than that of NAD6 and D6.

Chl content: It has been reported that COS with $\mathrm{Mw}$ of $2 \mathrm{kDa}$ increased $\mathrm{Chl}$ content of leaves and helped leaves be greener (Dzung et al. 2011). The total Chl contents of wheat seedlings visibly increased $(27.3-32.1 \%)$ by any COS regardless of different DA (Fig. 4). The Chl $a, b$, and $\mathrm{Chl}(a+b)$ of the D6 treatment group showed the highest 


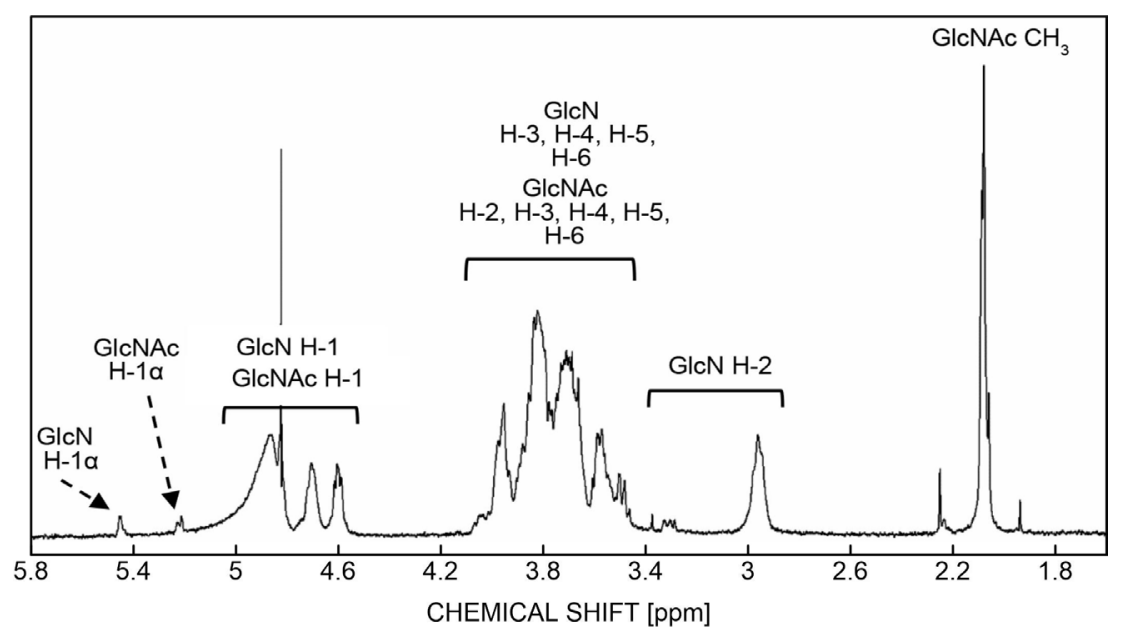

Fig. 1. ${ }^{1} \mathrm{H}$ NMR spectrum of partially $\mathrm{N}$-acetylated chitosan hexamer (NAD6). The assignment of peaks is deduced from literature (Trombotto et al. 2008).

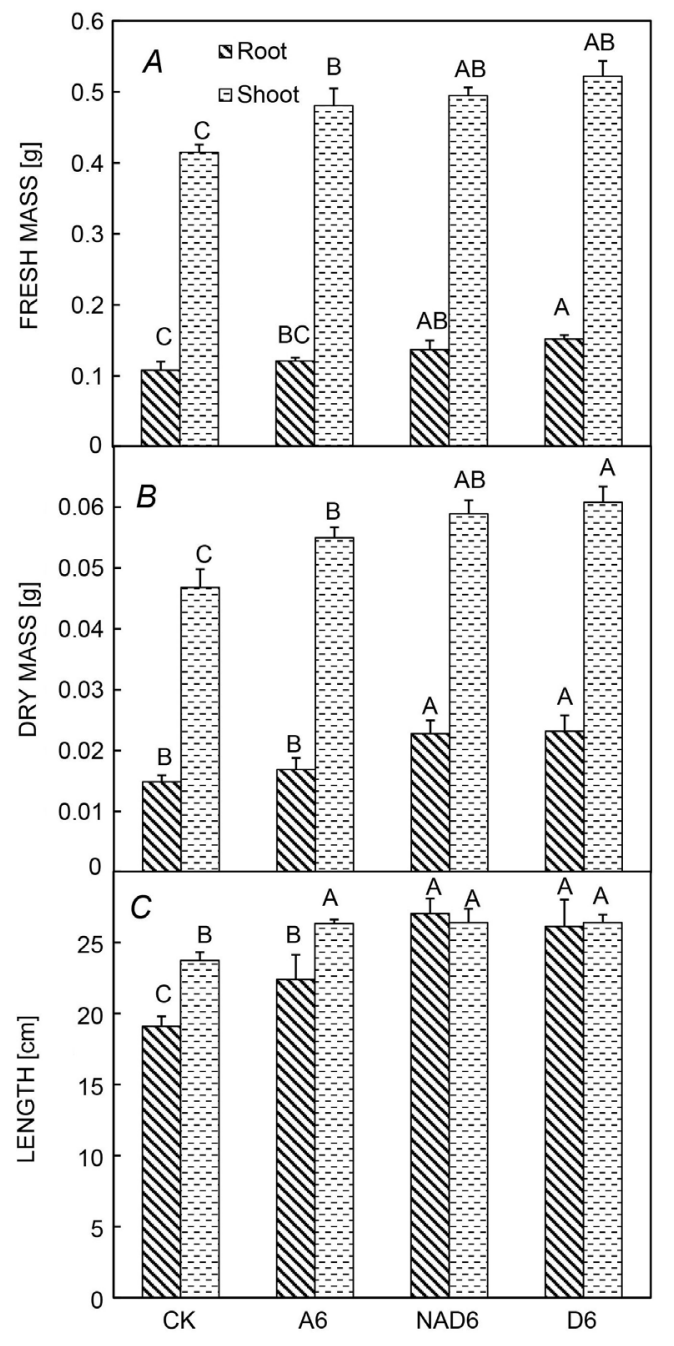

Fig. 2. Effects of three chitin and chitosan hexamers on growth and biomass accumulation of wheat seedlings. $(A)$ Fresh masses of root and shoot; $(B)$ dry masses of root and shoot; $(C)$ lengths of root and shoot. Values are the mean $\pm \mathrm{SD}$ of three independent replicates. Different letters indicate significant differences at $P<0.05$. CK - control; A6 - chitin hexamer; NAD6 - N-acetylated chitosan hexamer; D6 - chitosan hexamer.

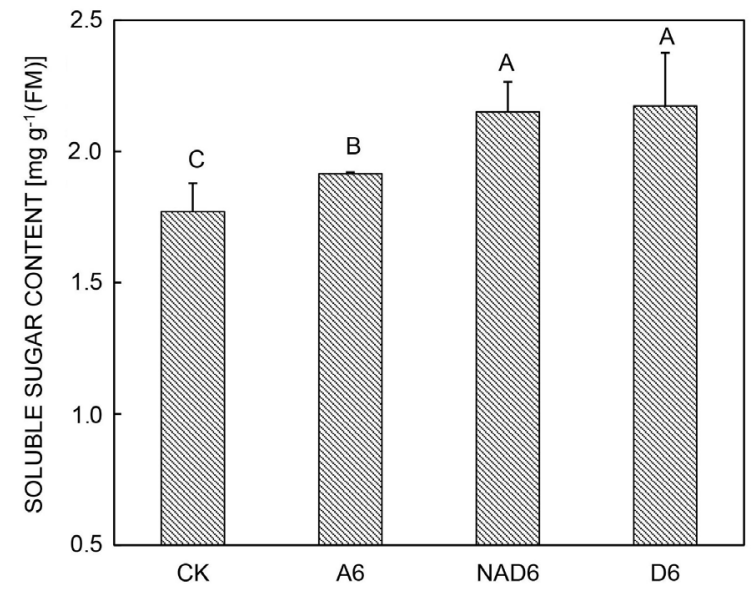

Fig. 3. Effects of three chitin and chitosan hexamers on soluble sugar content in leaves of wheat seedlings. Values are the mean \pm SD of three independent replicates. Different letters indicate significant differences at $P<0.05$. CK - control; A6 - chitin hexamer; NAD6 - N-acetylated chitosan hexamer; D6 - chitosan hexamer.

value but no obvious variation trend was observed among three hexamer-treated groups.

Photosynthetic characteristics: Table 1 showed the DA effects of chitin and chitosan hexamers on the photosynthesis of wheat seedlings. It seems that in this study all chitin and chitosan hexamers had no significant effect on the $C_{\mathrm{i}}$ values of the seedlings. However, two chitosan hexamers, D6 and NAD6, could increase the $E$ value of wheat seedlings by $31.1-57.5 \%$ relative to the control group. Furthermore, all chitin and chitosan hexamers could promote the value of $g_{\mathrm{s}}$, correspondingly resulting in much higher $P_{\mathrm{N}}$ values compared with the control group. After A6, NAD6, and D6 treatments, the values of $P_{\mathrm{N}}$ increased by $41.9,83.3$, and $90.0 \%$, respectively. The results indicate that more deacetylated chitosan hexamers are more effective fraction to promote photosynthesis of wheat seedlings. 
Chl fluorescence parameters: Three chitin and chitosan hexamers did not show promotion effect on $\Phi_{\mathrm{PSII}}$ and NPQ values compared with the control (Table 2). However, $\mathrm{F}_{\mathrm{v}} / \mathrm{F}_{\mathrm{m}}$ of wheat seedlings increased by the treatment of NAD6 and D6. Furthermore, D6 also increased $\mathrm{q}_{\mathrm{L}}$ value significantly. These results indicate D6 could improve the light-utilization efficiency of wheat seedlings.

Enzyme activity of Calvin cycle: Compared with the control plants, three oligosaccharides treatments led to markedly elevated activities of Rubisco in wheat leaves (Fig. 5). D6 showed the strongest promoting effect but no significant difference was observed between three oligosaccharides treatment groups. In contrast, the chitin and chitosan hexamers with various DA showed different

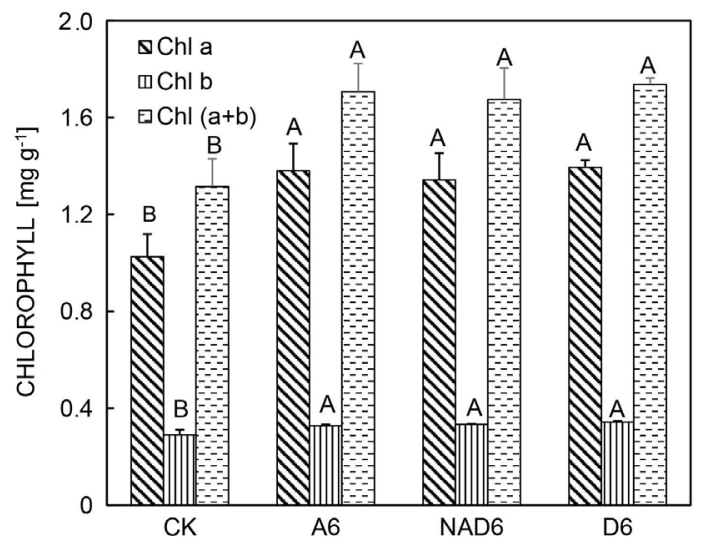

Fig. 4. Effects of three chitin and chitosan hexamers on chlorophyll contents of wheat seedlings. Values are the mean \pm $\mathrm{SD}$ of three independent replicates. Different letters indicate significant differences at $P<0.05$. CK - control; A6 - chitin hexamer; NAD6 - N-acetylated chitosan hexamer; D6 - chitosan hexamer. effects on FBPase activity. Compared with control group, A6, NAD6, and D6 increased the FBPase activities by $31.0,44.6$, and $80.6 \%$, respectively. The present results indicate the fully deacetylated chitosan hexamer showed a stronger promoting effect on the FBPase activity of wheat seedlings than other two acetylated hexamers.

\section{Discussion}

The bioactivities of COS are closely dependent on its chemical structure. Degree of polymerization (DP) and DA are both two key structural factors of COS and have attracted particular attention in the previous studies on the structure-function relationship of COS (Li et al. 2016). In agriculture, Kulikov et al. (2006) reported that five chitosan and $\operatorname{COS}$ fractions with average $\mathrm{Mw}$ varying from 1.2 to $40.4 \mathrm{kDa}$ could effectively suppress systemic infection of mild mosaic virus in bean (Phaseolus vulgaris L.) plants, and the degree of chitosan-induced antiviral resistance increased as the Mw decreased. Similarly, COSs with low $\mathrm{Mw}$ were recognized as being more active to promote plant growth and induce plant against abiotic stress compared to chitosan (Nge et al. 2006, Zong et al. 2017b). On the other hand, $\mathrm{N}$-acetyl group also plays an important role in the bioactivity of COSs for agricultural application. Yamada et al. (1993) reported that chitin oligosaccharides showed very high elicitor activity to induce the formation of phytoalexins in suspension-cultured rice cells, but their deacetylated form was inactive. Zou et al. (2015) investigated the ability of COSs with different DA to elicit wheat resistance against salt stress and found that the partially $\mathrm{N}$-acetylated COS with $50 \%$ DA showed the most potent activity. However, in previous experiments, COSs were mostly derived from chemical or enzymatic degradation of chitosan. Lots of COSs used for bioactivity assays were still a complex mixture, which were classified according to their average DA, DP, or Mw. Generally, when

Table 1. Effects of three chitin and chitosan hexamers on photosynthesis of wheat seedlings. Values are the mean \pm SD of three independent replicates. Different letters indicate significant differences at $P<0.05$. CK - control; A6 - chitin hexamer; NAD6 $\mathrm{N}$-acetylated chitosan hexamer; D6 - chitosan hexamer.

\begin{tabular}{lllll}
\hline & $C_{\mathrm{i}}\left[\mu \mathrm{mol} \mathrm{mol}^{-1}\right]$ & $E\left[\mathrm{mmol} \mathrm{m}^{-2} \mathrm{~s}^{-1}\right]$ & $g_{\mathrm{s}}\left[\mathrm{mol} \mathrm{m}^{-2} \mathrm{~s}^{-1}\right]$ & $P_{\mathrm{N}}\left[\mu \mathrm{mol} \mathrm{m}^{-2} \mathrm{~s}^{-1}\right]$ \\
\hline CK & $287.42 \pm 6.86^{\mathrm{a}}$ & $5.36 \pm 1.34^{\mathrm{b}}$ & $0.19 \pm 0.03^{\mathrm{b}}$ & $12.85 \pm 2.34^{\mathrm{c}}$ \\
A6 & $290.25 \pm 20.93^{\mathrm{a}}$ & $5.78 \pm 0.59^{\mathrm{b}}$ & $0.29 \pm 0.05^{\mathrm{a}}$ & $18.23 \pm 1.51^{\mathrm{b}}$ \\
NAD6 & $277.75 \pm 15.17^{\mathrm{a}}$ & $7.03 \pm 0.56^{\mathrm{a}}$ & $0.28 \pm 0.03^{\mathrm{a}}$ & $23.57 \pm 3.64^{\mathrm{ab}}$ \\
D6 & $286.00 \pm 6.59^{\mathrm{a}}$ & $8.44 \pm 1.29^{\mathrm{a}}$ & $0.37 \pm 0.09^{\mathrm{a}}$ & $24.42 \pm 4.05^{\mathrm{a}}$ \\
\hline
\end{tabular}

Table 2. Effects of three chitin and chitosan hexamers on chlorophyll fluorescence parameters of wheat seedlings. Values are the mean \pm SD of three independent replicates. Different letters indicate significant differences at $P<0.05$. CK - control; A6 - chitin hexamer; NAD6 - N-acetylated chitosan hexamer; D6 - chitosan hexamer.

\begin{tabular}{lllll}
\hline & $\Phi_{\text {PSII }}$ & $\mathrm{F}_{\mathrm{v}} / \mathrm{F}_{\mathrm{m}}$ & $\mathrm{q}_{\mathrm{L}}$ & $\mathrm{NPQ}$ \\
\hline $\mathrm{CK}$ & $0.63 \pm 0.02^{\mathrm{a}}$ & $0.80 \pm 0.01^{\mathrm{b}}$ & $1.23 \pm 0.00^{\mathrm{b}}$ & $0.36 \pm 0.03^{\mathrm{a}}$ \\
$\mathrm{A} 6$ & $0.63 \pm 0.03^{\mathrm{a}}$ & $0.80 \pm 0.01^{\mathrm{b}}$ & $1.22 \pm 0.15^{\mathrm{ab}}$ & $0.36 \pm 0.02^{\mathrm{a}}$ \\
$\mathrm{NAD6}$ & $0.63 \pm 0.01^{\mathrm{a}}$ & $0.81 \pm 0.01^{\mathrm{a}}$ & $1.26 \pm 0.04^{\mathrm{ab}}$ & $0.38 \pm 0.05^{\mathrm{a}}$ \\
D6 & $0.63 \pm 0.02^{\mathrm{a}}$ & $0.81 \pm 0.01^{\mathrm{a}}$ & $1.27 \pm 0.35^{\mathrm{a}}$ & $0.35 \pm 0.04^{\mathrm{a}}$
\end{tabular}




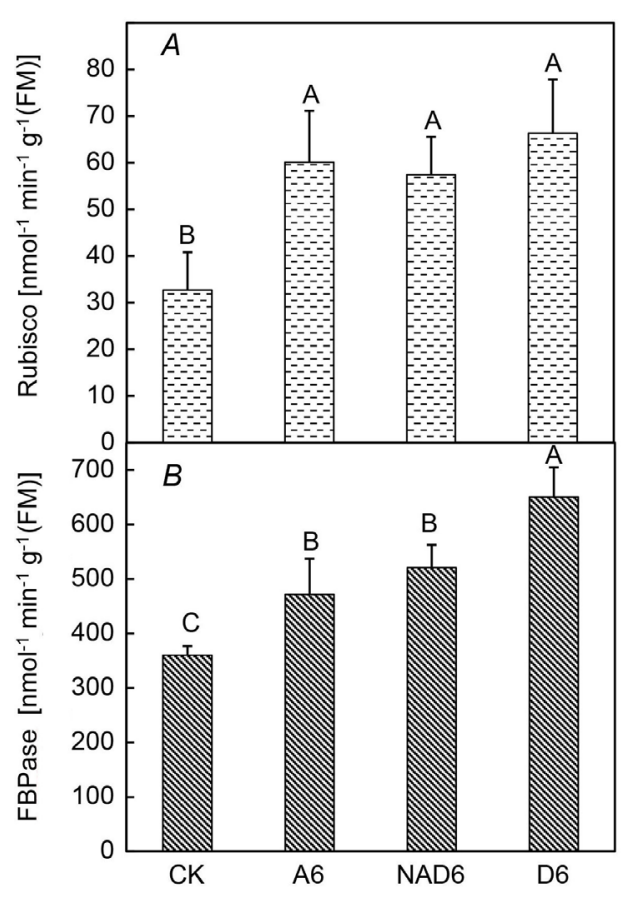

Fig. 5. Effects of three chitin and chitosan hexamers on enzyme activity of photosynthetic carbon metabolism in leaves of wheat seedlings. Each value represents the mean \pm SD of three independent replicates. Different letters indicate significant differences at $P<0.05$. (A) Rubisco, ribulose-1,5-bisphosphate carboxylase/oxygenase; $(B)$ FBPase, fructose-1,6-bisphosphatase. CK - control; A6 - chitin hexamer; NAD6 - N-acetylated chitosan hexamer; D6 - chitosan hexamer.

investigating the influence of COS with a certain property on its bioactivity, other parameters of COS should remain the same. However, it is actually difficult to control both DA and DP factors simultaneously using a COS mixture. Even the COSs with the same average DP and DA are used in the bioactivity assays, such as mixture of D2, D3, D4A2, and mixture of D1A1, D2A1, D6, which also may lead to controversial results. Therefore, the heterogeneous distribution of DA and DP in the COS mixtures would cause to some extent uncertainty on the structure-function relationship.

As for a fully deacetylated COS series (DA 0\%), chitosan hexamer (D6) was reported to be one of the best fragments promoting plant growth by our recent study (Zhang et al. 2016). In this case, a partially acetylated chitosan hexamer NAD6 with DA of $32.2 \%$ was further prepared by a reacetylation reaction. In combination with the commercialized chitin hexamers (DA 100\%), three COSs with identical DP and various DA were employed to investigate the effects of DA on its activity of promoting plant growth. The results suggested that chitosan hexamer exhibited a stronger effect than that of chitin hexamer, indicating that $\mathrm{N}$-acetylation might not be indispensable for promoting plant growth of COS. The shoot length of all three hexamer groups had no significant difference. However, the root length of NAD6 and D6 groups was obviously higher than that of the chitin hexamer group.
Root system can anchor the overground part of plant, and also acts as a highly elaborate absorptive organ enabling nutrients to be absorbed and transported to the rest of the plant. Therefore, a well-developed root system is essential for crop growth (González-Pérez et al. 2012). As a plant growth regulator, chitosan hexamers could promote the root growth, resulting in the whole plant biomass accumulation. These results were also consistent with the data of the soluble sugar content (Fig. 3).

Net photosynthetic rate $\left(P_{\mathrm{N}}\right)$, stomatal conductance $\left(g_{\mathrm{s}}\right)$, and transpiration rate $(E)$ are potential parameters to evaluate wheat cultivar performance (Subrahmanyam et al. 2006). Li et al. (2008) has reported that COS treatments could promote photosynthetic parameters of Brassica napus. In accordance with this study, our results showed in Table 1 indicated that all three COS samples could improve the $g_{\mathrm{s}}, E$, and $P_{\mathrm{N}}$ of wheat seedlings, and compared with chitin hexamer, chitosan hexamers showed more effective on $P_{\mathrm{N}}$ promotion. The $\mathrm{Chl}$ fluorescence results also indicate the light-utilization efficiency of wheat seedlings could be significantly improved by the full deacetylated chitosan fragments (Table 2). It seemed that $\mathrm{N}$-acetylation was not a structurally essential for the improvement of photosynthesis, which was further proved by the results of enzyme assay of photosynthetic carbon fixation (Calvin cycle) (Fig. 5). Overall, fully deacetylated COS was more effective to promote photosynthetic carbon fixation and generate more carbohydrate products for plant growth.

$\mathrm{N}$-acetyl group of chitin and chitosan oligomers was reported to play a vital role in binding with its receptor and eliciting plant resistance (Liu et al. 2012); however, our results suggested fully deacetylated chitosan hexamer showed the stronger effect on promoting plant growth than that of chitin hexamer. With regard to partially acetylated chitosan hexamer, its promoting growth activity was in an intermediate level but closer to the fully deacetylated chitosan hexamer. Considering that NAD6 has a relatively low DA (32.2\%), it is plausible that the principal promoting effect observed in this study was a consequence of a response to fragments with lesser $\mathrm{N}$-acetylation. Therefore, in this case of promoting plant growth, the role of $\mathrm{N}$-acetylation is different from those previous studies about elicitor activity of chitin and chitosan oligomers (Liu et al. 2012, Zou et al. 2015). Different performance between chitin oligomers as elicitors and plant growth promoters was also presented in those reports on molecular size effects. Shibuya group proved that chitin oligosaccharides smaller than triose showed negligible elicitor activity (Yamada et al. 1993, Shibuya et al. 1996), but Winkler et al. (2017) recently reported these shortchain chitin oligomers were very effective to stimulate plant growth. These results suggested that the elicitor activity and promoting growth of chitin/chitosan-derived compounds in plants are supposed to depend on different signaling pathways.

In the past few years, numerous pioneering works for identifying the high-affinity binding protein of chitin oligomers in various plants were carried out and the signal recognition of chitin involved in elicitor activity are 
getting clearer (Liu et al. 2012, Hayafune et al. 2014). On the other hand, concerning the signal pathway of chitosan in plants, whether its specific receptors exist in plants or not is still in dispute (Yin et al. 2016). Chitosan oligomers with one amino group on $\mathrm{C} 2$ are cationic oligosaccharides, which might be attracted to negative plant cell walls (Cabrera et al. 2010). This property makes the function and mechanism of chitosan oligomers more complicated. Regarding the promoting growth of plants in response to chitin and chitosan oligomers in natural conditions, metabolite profiles and transcriptomics have been performed, revealing a pleiotropic modulation of carbon and nitrogen metabolism of plants induced by chitin and chitosan oligomers (Zhang et al. 2017b, 2018; Winkler et al. 2017). However, the exact molecular mechanism of promoting plant growth is not completely unraveled and needs more biochemical clarification.

Conclusions: The present study investigated the effect of exogenous chitin and chitosan hexamers with different DA on growth promotion of wheat seedlings. We concluded following: (1) All chitin and chitosan hexamers could significantly enhance the growth and photosynthesis of wheat seedlings. (2) Two chitosan hexamers were more effective in enhancing plant growth and photosynthesis than chitin hexamer, and homogeneous $(\mathrm{GlcN})_{6}$ seemed to be the optimal among the three samples, revealing $\mathrm{N}$-acetylation may not be indispensable in promoting plant growth of COS. These results would be instructive for the development of high-efficient biostimulator of COS and expand its application in the field of agriculture.

\section{References}

Cabrera J.C., Boland A., Cambier P. et al.: Chitosan oligosaccharides modulate the supramolecular conformation and the biological activity of oligogalacturonides in Arabidopsis. Glycobiology 20: 775-786, 2010.

Chatelain P.G., Pintado M.E., Vasconcelos M.W.: Evaluation of chitooligosaccharide application on mineral accumulation and plant growth in Phaseolus vulgaris. - Plant Sci. 215-216: 134-140, 2014.

Domard A.: A perspective on 30 years research on chitin and chitosan. - Carbohydr. Polym. 84: 696-703, 2011.

Dzung N.A., Khanh V.T.P., Dzung T.T.: Research on impact of chitosan oligomers on biophysical characteristics, growth, development and drought resistance of coffee. - Carbohydr. Polym. 84: 751-755, 2011.

Dzung P.D., Phu D.V., Du B.D. et al.: Effect of foliar application of oligochitosan with different molecular weight on growth promotion and fruit yield enhancement of chili plant. - Plant Prod. Sci. 20: 389-395, 2017.

Fernandes J.C., Spindola H., de Sousa V. et al.: Anti-inflammatory activity of chitooligosaccharides in vivo. - Mar. Drugs 8: 1763-1768, 2010.

González-Pérez L., Vázquez-Glaría A., Perrotta L. et al.: Oligosaccharins and Pectimorf ${ }^{\mathbb{B}}$ stimulate root elongation and shorten the cell cycle in higher plants. - Plant Growth Regul. 68: 211-221, 2012.

Hayafune M., Berisio R., Marchetti R. et al.: Chitin-induced activation of immune signaling by the rice receptor CEBiP relies on a unique sandwich-type dimerization. - P. Natl. Acad. Sci. USA. 111: E404-E413, 2014.
Huang R., Mendis E., Rajapakse N., Kim S.K.: Strong electronic charge as an important factor for anticancer activity of chitooligosaccharides (COS). - Life Sci. 78: 2399-2408, 2006.

Hurry V.M., Keerberg O., Pärnik T. et al:: Cold-hardening results in increased activity of enzymes involved in carbon metabolism in leaves of winter rye (Secale cereale L). Planta 195: 554-562, 1995.

Jabeen N., Ahmad R.: The activity of antioxidant enzymes in response to salt stress in safflower (Carthamus tinctorius L.) and sunflower (Helianthus annuus L.) seedlings raised from seed treated with chitosan. - J. Sci. Food Agr. 93: 1699-1705, 2013.

Kananont N., Pichyangkura R., Chanprame S. et al.: Chitosan specificity for the in vitro seed germination of two Dendrobium orchids (Asparagales: Orchidaceae). - Sci. Hortic.-Amsterdam 124: 239-247, 2010.

Khan W.M., Prithiviraj B., Smith D.L.: Effect of foliar application of chitin and chitosan oligosaccharides on photosynthesis of maize and soybean. - Photosynthetica 40: 621-624, 2002.

Kuchitsu K., Kosaka H., Shiga T., Shibuya N.: EPR evidence for generation of hydroxyl radical triggered by $N$-acetylchitooligosaccharide elicitor and a protein phosphatase inhibitor in suspension-cultured rice cells. - Protoplasma 188: 138-142, 1995.

Kulikov S.N., Chirkov S.N., Il'ina A.V. et al.: Effect of the molecular weight of chitosan on its antiviral activity in plants. - Appl. Biochem. Microbiol. 42: 200-203, 2006.

Kumar A.B.V., Varadaraj M.C., Gowda L.R., Tharanathan R.N.: Characterization of chito-oligosaccharides prepared by chitosanolysis with the aid of papain and Pronase, and their bactericidal action against Bacillus cereus and Escherichia coli. - Biochem. J. 391: 167-175, 2005.

Li K., Liu S., Xing R. et al.: High-resolution separation of homogeneous chitooligomers series from 2-mers to 7-mers by ion-exchange chromatography. - J. Sep. Sci. 36: 1275-1282, 2013.

Li K., Xing R., Liu S. et al.: Access to N-acetylated chitohexaose with well-defined degrees of acetylation. - Biomed Res. Int. 2017: 2486515, 2017.

Li K., Xing R., Liu S., Li P.: Advances in preparation, analysis and biological activities of single chitooligosaccharides. Carbohydr. Polym. 139: 178-190, 2016.

Li Y., Zhao X., Xia X. et al.: [Effects of oligochitosan on photosynthetic parameters of Brassica napus seedlings under drought stress.] - Acta Agron. Sin. 34: 326-329, 2008. [In Chinese]

Lichtenthaler H.K., Wellburn A.R.: Determinations of total carotenoids and chlorophylls $a$ and $b$ of leaf extracts in different solvents. - Biochem. Soc. T. 11: 591-592, 1983.

Lieder R., Thormodsson F., Ng C.H. et al.: Chitosan and chitin hexamers affect expansion and differentiation of mesenchymal stem cells differently. - Int. J. Biol. Macromol. 51: 675-680, 2012.

Liu T., Liu Z., Song C. et al.: Chitin-induced dimerization activates a plant immune receptor. - Science 336: 1160-1164, 2012.

Muzzarelli R.A.A.: Chitins and chitosans as immunoadjuvants and non-allergenic drug carriers. - Mar. Drugs 8: 292-312, 2010.

Nge K.L., Nwe N., Chandrkrachang S., Stevens W.F.: Chitosan as a growth stimulator in orchid tissue culture. - Plant Sci. 170: 1185-1190, 2006.

Rinaudo M.: Chitin and chitosan: Properties and applications. Prog. Polym. Sci. 31: 603-632, 2006.

Roháček K.: Chlorophyll fluorescence parameters: the defini- 
tions, photosynthetic meaning, and mutual relationships. Photosynthetica 40: 13-29, 2002.

Sharkey T.D., Savitch L.V., Butz N.D.: Photometric method for routine determination of $\mathrm{k}_{\text {cat }}$ and carbamylation of Rubisco. Photosynth. Res. 28: 41-48, 1991.

Shibuya N., Ebisu N., Kamada Y. et al.: Localization and binding characteristics of a high-affinity binding site for $\mathrm{N}$-acetylchitooligosaccharide elicitor in the plasma membrane from suspension-cultured rice cells suggest a role as a receptor for the elicitor signal at the cell surface. - Plant Cell Physiol. 37: 894-898, 1996.

Subrahmanyam D., Subash N., Haris A., Sikka A.K.: Influence of water stress on leaf photosynthetic characteristics in wheat cultivars differing in their susceptibility to drought. Photosynthetica 44: 125-129, 2006.

Trombotto S., Ladavière C., Delolme F., Domard A.: Chemical preparation and structural characterization of a homogeneous series of chitin/chitosan oligomers. - Biomacromolecules 9: 1731-1738, 2008.

Vander P., Vårum K.M., Domard A. et al.: Comparison of the ability of partially $\mathrm{N}$-acetylated chitosans and chitooligosaccharides to elicit resistance reactions in wheat leaves. - Plant Physiol. 118: 1353-1359, 1998.

Wang M., Chen Y., Zhang R. et al.: Effects of chitosan oligosaccharides on the yield components and production quality of different wheat cultivars (Triticum aestivum L.) in Northwest China. - Field Crop. Res. 172: 11-20, 2015.

Wang Z., Zheng L., Yang S. et al.: N-acetylchitooligosaccharide is a potent angiogenic inhibitor both in vivo and in vitro. Biochem. Bioph. Res. Co. 357: 26-31, 2007.

Winkler A.J., Dominguez-Nuñez J.A., Aranaz I. et al:: Shortchain chitin oligomers: Promoters of plant growth. - Mar. Drugs 15: 40, 2017.

Xing R., Liu Y., Li K. et al.: Monomer composition of chitooligosaccharides obtained by different degradation methods and their effects on immunomodulatory activities. -
Carbohydr. Polym. 157: 1288-1297, 2017.

Yamada A., Shibuya N., Kodama O., Akatsuka T.: Induction of phytoalexin formation in suspension-cultured rice cells by $\mathrm{N}$-acetylchitooligosaccharides. - Biosci. Biotech. Bioch. 57: 405-409, 1993.

Yin H., Du Y., Dong Z.: Chitin oligosaccharide and chitosan oligosaccharide: Two similar but different plant elicitors. Front. Plant Sci. 7: 522, 2016.

Zhang X., Li K., Liu S. et al.: Size effects of chitooligomers on the growth and photosynthetic characteristics of wheat seedlings. - Carbohydr. Polym. 138: 27-33, 2016.

Zhang X., Li K., Liu S. et al.: Relationship between the degree of polymerization of chitooligomers and their activity affecting the growth of wheat seedlings under salt stress. - J. Agr. Food Chem. 65: 501-509, 2017a.

Zhang X., Li K., Xing R. et al.: Metabolite profiling of wheat seedlings induced by chitosan: Revelation of the enhanced carbon and nitrogen metabolism. - Front. Plant Sci. 8: 2017, $2017 \mathrm{~b}$.

Zhang X., Li K., Xing R. et al.: miRNA and mRNA expression profiles reveal insight into chitosan-mediated regulation of plant growth. - J. Agr. Food Chem. 66: 3810-3822, 2018.

Zong H., Li K., Liu S. et al.: Improvement in cadmium tolerance of edible rape (Brassica rapa L.) with exogenous application of chitooligosaccharide. - Chemosphere 181: 92-100, 2017a.

Zong H., Liu S., Xing R. et al.: Protective effect of chitosan on photosynthesis and antioxidative defense system in edible rape (Brassica rapa L.) in the presence of cadmium. - Ecotox. Environ. Safe. 138: 271-278, $2017 \mathrm{~b}$.

Zou P., Li K., Liu S. et al.: Effect of chitooligosaccharides with different degrees of acetylation on wheat seedlings under salt stress. - Carbohydr. Polym. 126: 62-69, 2015.

Zou P., Tian X., Dong B., Zhang C.: Size effects of chitooligomers with certain degrees of polymerization on the chilling tolerance of wheat seedlings. - Carbohydr. Polym. 160: 194202, 2017.

(C) The authors. This is an open access article distributed under the terms of the Creative Commons BY-NC-ND Licence. 